\title{
A RELATIVISTIC POLARON WITHOUT CUTOFFS IN TWO SPACE DIMENSIONS
}

\author{
BY ALAN SLOAN
}

Communicated by Peter D. Lax, November 17, 1971

I. Introduction. A model of a physical system in quantum field theory is heuristically given as a densely defined bilinear form on a Hilbert space $\mathscr{K}$. An important goal is to determine if the system is Hamiltonian. That is, does there exist a selfadjoint operator on some Hilbert space whose unitary group determines the dynamics of the system? A standard procedure is to smooth the bilinear form into a densely defined selfadjoint operator on $\mathscr{K}$ by introducing cutoffs. As the cutoffs are removed one attempts to prove that the resulting sequence of operators converges. In physically realistic cases, this limiting operator, the renormalized Hamiltonian, if it exists at all, can only be defined on a new Hilbert space, $\mathscr{K}_{\text {ren }}$, orthogonal to the original $\mathscr{K} . \mathscr{K}_{\text {ren }}$ is the space of physical states and may arise abstractly through the use of $C^{*}$-algebra techniques. Cases which approximate realistic models and in which $\mathscr{K}=\mathscr{K}_{\text {ren }}$ are useful because the familiar nature of $\mathscr{K}$ permits a detailed investigation of relevant questions, [1], [3], [6].

Nelson [8] examined a model of a nonrelativistic spinless nucleon interacting with relativistic neutral scalar mesons in three space dimensions via a Yukawa interaction in which the pair creation and annihilation terms had been dropped. He was able to define a renormalized Hamiltonian on $\mathscr{K}_{\text {ren }}=\mathscr{K}=\mathscr{K}\left(R^{3}\right)=L^{2}\left(R^{3}\right) \otimes \mathscr{F}\left(R^{3}\right)$ where $\mathscr{F}\left(R^{3}\right)$ is a symmetric Fock space over $L^{2}\left(R^{3}\right)$. That is, $\mathscr{F}\left(R^{3}\right)=\sum_{s=0}^{\infty} \mathscr{F}_{s}\left(R^{3}\right), \mathscr{F}_{0}\left(R^{3}\right)=$ complex numbers, $\mathscr{F}_{s}\left(R^{3}\right)=$ the $s$-fold symmetric tensor product of $L^{2}\left(R^{3}\right)$. As is pointed out in [8], the methods used are apparently limited to the case considered and do not apply if the nucleon has relativistic kinetic energy function. In fact, in this case, one must change Hilbert spaces [5].

The main result of this paper is that if we replace $R^{3}$ by $R^{2}$ and use the relativistic kinetic energy function for the nucleon, then it is possible to define a renormalized Hamiltonian on $\mathscr{K}\left(R^{2}\right)$. The methods we employ differ from those of [8]. No special dressing transformations are needed. We now make these remarks more explicit.

II. The polaron with cutofis. The 1-particle momentum state space for a 2-dimensional spinless electron or for a neutral scalar phonon is $L^{2}\left(R^{2}\right)$.

AMS 1970 subject classifications. Primary 81A18; Secondary 81A09, 81A19, 81A06. Key words and phrases. Polaron, cutoffs, quantum field theory, Nelson's model. 
$\mathscr{K}\left(R^{2}\right)$ is the state space for an electron and many phonons. In this paper we prove it is also the state space for a physical polaron. For any $m>0$, let $E_{m}(p)=\left(|p|^{2}+m^{2}\right)^{1 / 2}$. When $m=\theta=$ mass of the electron, multiplication by $E_{\theta}$ on $L^{2}\left(R^{2}\right)$ defines a selfadjoint operator, $H_{1}(\theta)$. When $m=\mu$ = mass of the phonon, the quantization [2, p. 223] of $H_{1}(\mu)$ defines a selfadjoint operator $\mathrm{H}_{2}$ on $\mathscr{F}\left(R^{2}\right)$.

Let $\mathscr{F}_{m}^{\prime}\left(R^{2}\right)$ denote the subspace of $\mathscr{F}_{m}\left(R^{2}\right)$ consisting of functions which are continuous in each variable separately. If $f$ is in $\mathscr{F}_{m}^{\prime}$ then for almost every $k$ in $R^{2}$, the function $f_{k}$ defined on $\left(R^{2}\right)^{m-1}$ by $f_{k}\left(k_{1}, \ldots, k_{m-1}\right)$ $=f\left(k_{1}, \ldots, k_{m-1}, k\right)$ is in $\mathscr{F}_{m-1}$. Define $a_{m}(k)$ to be the operator from $\mathscr{F}_{m}$ to $\mathscr{F}_{m-1}$ with domain $D\left(a_{m}(k)\right)=\left\{f \in \mathscr{F}_{m}^{\prime}: k \rightarrow f_{k}\right.$ is continuous from $\left.R^{2} \rightarrow \mathscr{T}_{m-1}\right\}$, and with action on that domain given by $a_{m}(k) f=m^{1 / 2} f_{k}$. For $m=0$ let $a_{m}(k)$ be the zero operator on $\mathscr{F}_{0}$. Let $a(k)$ be the operator on Tiven as the direct sum $\sum_{m=0}^{\infty} a_{m}(k)$. The phonon pointwise annihilation operator of momentum $k, a(k)$, is densely defined but not closed. Let $S_{4}$ denote translation on $L^{2}\left(R^{2}\right)$ by $q \in R^{2}$; i.e., $\left(S_{q} g\right)(p)=g(p-q)$ for $g$ in $L^{2}$ and $p$ in $R^{2}$. For each $f$ in $L^{2}\left(R^{2}\right)$ there is a unique bounded operator $V_{s}(f): L^{2} \otimes \mathscr{F}_{s} \rightarrow L^{2} \otimes \mathscr{F}_{s-1}$ such that for $g$ in $L^{2}$ and $h$ in $D\left(a_{s}(k)\right)$,

$$
V_{s}(f)(g \otimes h)=\int f(k)\left(S_{-k} g \otimes a_{s}(k) h\right) d k
$$

where the integral is strong. Let $V(f)=\sum_{s=0}^{\infty} V_{s}(f) .\left(V(f)+V(f)^{*}\right)^{* *}$ $=H_{I}(f)$ is a selfadjoint operator, relatively bounded with respect to $J(\theta)=H_{1}(\theta) \otimes I+I \otimes H_{2}$ and has relative bound zero. Consequently, $H(f ; \theta)=J(\theta)+H_{I}(f)$ is selfadjoint and bounded below and is the Hamiltonian for the 2-dimensional polaron with momentum cutoff $f$.

Let $P_{i}^{\prime}$ be multiplication by the $i$ th coordinate function, $i=1,2$. Let $P_{i}$ be the quantization of $P_{i}^{\prime}$. There is [4] a unitary operator $W: \mathscr{K}\left(R^{2}\right)$ $\rightarrow L^{2}\left(R^{2}, \mathscr{F}\left(R^{2}\right)\right)$ such that for any $h$ in $W$ (domain $\left.(J(\theta))\right)$ we have the formula $\left(W H(f ; \theta) W^{-1} h\right)(p)=H_{2} h(p)+E\left(p-\left(P_{1}, P_{2}\right)\right) h(p)$ $+\left(A(f)+A^{*}(f)\right)^{* *} h(p)$ for almost every $p$ in $R^{2} . A(f)$ is the phonon annihilation operator, [2, p. 235]. The total momentum decomposition $W$ is independent of $f$ and $\theta$.

Let $H_{0}(f ; \theta)=H_{2}+E_{\theta}\left(-\left(P_{1}, P_{2}\right)\right)+\left(A^{*}(f)+A(f)\right)^{* *} . H_{0}(f ; \theta)$ is a selfadjoint operator which is bounded below and represents the action of the Hamiltonian on "states" of zero total momentum. If $f$ is a real radial $L^{2}$ function then $\inf \left(\operatorname{spectrum}\left(H_{0}(f ; \theta)\right)\right)$ is an eigenvalue of multiplicity 1 and $\inf \left(\operatorname{spec}\left(H_{0}(f ; \theta)\right)\right)=\inf (\operatorname{spec}(H(f ; \theta)))$, [4]. Let $h(f ; \theta)$ be an eigenvector of norm $1 . h(f ; \theta)$ is a rest state of zero total momentum.

III. Removing the cutoff. The object we would like to consider is $H\left(E_{\mu}^{-1 / 2} ; \theta\right)$ which ought to be the Hamiltonian for the physical polaron. However, since $E_{\mu}^{-1 / 2} \notin L^{2}\left(R^{2}\right), V\left(E_{\mu}^{-1 / 2}\right)$ can only be defined on $\{0\}$. Never- 
theless, $V\left(E_{\mu}^{-1 / 2}\right)$ is a densely defined bilinear form and consequently so is $H\left(E_{\mu}^{-1 / 2} ; \theta\right)$. This is the form mentioned in the Introduction.

Let $g$ be a real $C^{\infty}$ function on $R^{1}$ that is 1 on $\left(-\frac{1}{2}, \frac{1}{2}\right)$, has range in $[0,1]$, and vanishes outside of $[-1,1]$. For every $m>0$ let $f_{m}(k)=E_{\mu}^{-1 / 2}(k)$ if $|k|<m$ and for $|k| \geqq m$, let $f_{m}(k)=E_{\mu}^{-1 / 2}(k) g\left(\frac{1}{4}+|k|-m\right)$. For each sufficiently large $m$, there is a $\theta_{m}$ such that $\inf \left(\operatorname{spec}\left(H\left(f_{m} ; \theta_{m}\right)\right)\right)=0 . H_{m}$ $=H\left(f_{m} ; \theta_{m}\right)$ is the Hamiltonian with cutoff $f_{m}$ after mass renormalization. Let $h_{m}=h\left(f_{m} ; \theta_{m}\right)$.

THEOREM. There is a nonnegative, selfadjoint operator, $H_{\infty}$, densely defined on $\mathscr{K}\left(R^{2}\right)$ such that strong limit $f\left(H_{m}\right)=f\left(H_{\infty}\right)$ as $m \rightarrow \infty$ where either (1) $f(x)=(x+r)^{-1}, r>0$; or (2) $f(x)=e^{i t x}$, t real; or (3) $f(x)=e^{-t x}$, $t \geqq 0$, for some sequence $\{m\}$.

The proof of (2) and (3) follows directly from (1), [7, p. 502]. The proof of (1) depends on the fact that $\left\{h_{m}\right\}_{m=0}^{\infty}$ lies in a norm compact subset of $\mathscr{F}\left(R^{2}\right)$. This in turn depends on the estimate $\left\|a\left(k_{1}\right) \ldots a\left(k_{t}\right) h_{m}\right\| \leqq \prod_{i=1}^{t} E_{\mu}^{-3 / 2}\left(k_{i}\right)$, first derived informally by Gross [5], using the nonnegativity of $H_{n}$ and the commutation relations of $H_{n}$ with $a(k)$. Similar techniques show that the maps $k \rightarrow a(k) h_{m}$ are continuous with compact support and have partial derivatives in coordinate directions which are uniformly bounded on compact sets. Consequently the finite particle components of $h_{m}$ have similar properties. If $N_{\tau}, 1>\tau>0$, denotes the quantization of multiplication by $E_{\mu}^{\tau}$ on $L^{2}\left(R^{2}\right)$, then the above estimate shows that $\sup _{m}\left\|\left(N_{\tau}\right)^{1 / 2} h_{m}\right\|<\infty$. If $h_{\infty}$ is any limit point of $\left\{h_{m}\right\}$, it also follows that $h_{\infty}$ is an entire vector for the total number of phonons operator and is in the domain of $N_{\tau}^{1 / 2}$. Also $\left(h_{\infty}, 1\right)>0$ where $1 \in \mathscr{F}_{0}$.

For $\alpha$ any $C^{\infty}$ function on $R^{2}$ with sufficiently small support about the origin define $h_{\alpha}$ in $L^{2}\left(R^{2}, \mathscr{F}\right)$ by $h_{\alpha}(p)=\alpha(p) h_{\infty}$. Applying an irreducible algebra of operators to $h_{\alpha}$ yields a dense subset on which $\left\{\left(H_{m}+r\right)^{-1} W^{-1}\right\}$ converges strongly for all $r>0$ and some subsequence $\{m\}$ of $\{1,2, \ldots\}$. Let $R(r)$ be the strong limit. To show it is the resolvent of a nonnegative selfadjoint operator we need only prove the null space of $R(r)$ is 0 . This follows from the fact that strong $\lim _{r \rightarrow \infty} r R(r)=$ Identity. $^{1}$

\section{BiBLIOGRAPHY}

1. J. Cannon, Quantum field theoretic properties of a model of Nelson: Domain and eigenvector stability for perturbed linear operators, J. Functional Analysis 8 (1971), 101-152.

2. J. Cook, The mathematics of second quantization, Trans. Amer. Math. Soc. 74 (1953), 222-245. MR 14, 825.

3. J. P. Eckmann, A model with persistent vacuum, Comm. Math. Phys. 18(1970), 247-264.

4. L. Gross, Existence and uniqueness of physical ground states, Cornell University, Ithaca, New York, 1970 (preprint).

\footnotetext{
${ }^{1}$ Detailed proofs of the results announced here can be found in the author's doctoral dissertation, written under Leonard Gross at Cornell University during 1971, who suggested many of the techniques there employed.
} 
5. _- Relativistic polaron without cutoffs, Cornell University, Ithaca, New York, 1971 (preprint).

6. R. Høegh-Krohn, Asymptotic fields in some models of quantum field theory. III, J. Mathematical Phys. 11 (1970), 185-188. MR 41 \# 3035.

7. T. Kato, Perturbation theory for linear operators, Die Grundlehren der math. Wissenschaften, Band 132, Springer-Verlag, New York, 1966. MR 34 \# 3324.

8. E. Nelson, Interaction of nonrelativistic particles with a quantized scalar field, J. Mathematical Phys. 5 (1964), 1190-1197. MR 30 \# 5721.

Department of Mathematics, Carnegie-Mellon University, Pittsburgh, PennsylVANIA 15213 